\title{
Different steady states for spin currents in noncollinear multilayers
}

\author{
Peter M Levy and Jianwei Zhang \\ Department of Physics, New York University, New York, NY,10003,USA \\ 21 November 2018
}

PACS numbers: 72.25.-b,72.15.Gd, 73.23.-b

\begin{abstract}
We find there are at least two different steady states for transport across noncollinear magnetic multilayers. In the conventional one there is a discontinuity in the spin current across the interfaces which has been identified as the source of current induced magnetic reversal; in the one advocated herein the spin torque arises from the spin accumulation transverse to the magnetization of a magnetic layer. These two states have quite different attributes which should be discerned by current experiments.
\end{abstract}


Recent interest has focused on electron transport across metallic multilayers which contain the $3 \mathrm{~d}$ transition metals, especially the spin torque acting on a magnetic layer as current is driven across noncollinear magnetic multilayers. [1] To calculate the transport properties of multilayered structures one would ideally calculate the wavefunctions for the entire structure and then evaluate the conductivity in the presence of impurities; [2] in this approach one retains the long range nature of the conductivity, which is important even for diffusive metals. [3] However what is usually done is to adopt a layer-by-layer approach in which one solves for the transport within homogeneous layers and connects the out of equilibrium distribution functions with transmission and reflection coefficients at the interfaces. These functions are statistical density matrices and contain less information than wavefunctions, therefore one should anticipate some loss of information that may alter the conductivity one calculates for the entire layered structure. Indeed this has been found when discussing giant magnetoresistance, and it was shown that it necessary to include either spin dependent electrochemical potentials or equivalently spin accumulation in the layer-by-layer approach which one would not explicitly do if one determined the wavefunctions for the entire approach. 4] Most of the analyses of transport in multilayers has been done on collinear structures and as far as one can tell the use of spin dependent electric fields or accumulation has been able to make up for the putative shortcomings of the layer-by layer approach for the conductivity of the whole structure. However when one studies noncollinear magnetic multilayers the previous constructs used to shore up the layer-by-layer approach may well be insufficient and, unless augmented by additional artifices, can lead to erroneous predictions, e.g., as to the microscopic origin of spin torque. Here we present the case for adding such a new construct: current induced contributions to the transmission coefficients which connect the out of equilibrium distribution functions between layers.

When current is driven across a magnetically multilayered structure the first response of the itinerant carriers is a rapid accumulation of charge around the interfaces to establish a steady state charge current, i.e., one that does not vary with time; in metallic structures this accumulation is confined to a screening length of the order of angströms. The difference in the density of states at the Fermi level and scattering rates for spin up/down electrons leads to a current which is spin polarized, i.e., $j_{\uparrow}-j_{\downarrow} \neq 0$ in the magnetic layers, while the current in the normal layers is not polarized. This discontinuity in the polarized current at interfaces between ferromagnetic and normal metal layers $(\mathrm{N} / \mathrm{F})$ is the source of spin accumulation about the interface; its length scale, known as the spin diffusion length, is controlled by the rate of spin flip $\tau_{s f}^{-1}$ in the layers; it is only after one has established this spin accumulation that the spin polarized current from one magnetic layer can be transmitted to another provided that the normal (non magnetic) spacer layer is less than the spin diffusion length. [5] It is also true that one achieves a steady state spin current after $t \gtrsim \tau_{s f}$ which is of the order of a picosecond, say in $\mathrm{Cu}$; conversely if one does not consider the source term at interfaces there is no accumulation and no steady state in the thermodynamic sense, i.e., one cannot achieve a state of maximum entropy production. 6] 
For collinear structures when the spin accumulation created at one N/F interface arrives at the other interface of the normal layer it is superimposed on the polarization that exists in equilibrium; while it alters the interface scattering potential at the second interface it does not contribute in linear response, rather it is a nonlinear effect. It is for this reason that one uses the transmission coefficients found from equilibrium band structure calculations. However for noncollinear structures the spin polarization that is superimposed has a component that is transverse to the polarization of the second interface. This does not lead to a current driven repopulation of equilibrium states ,i.e., additional (nonlinear) accumulation, but to a "current induced spin flip" between states of opposite spin; this has been recently shown to lead to a new contribution to the transmission coefficient that enters in linear response. [7] We have found that this additional term makes the difference in the predictions based on models which use realistic band structures for the individual layers and equilibrium transmission coefficients to connect the nonequilibrium distribution functions across interfaces, 8 and those which use free electron bands which maintain spin coherence across the layers and mimic the scattering at interfaces by phenomenological interface resistances, 9] i.e., when one includes the additional interface scattering in the former models one retrieves the features found in the latter.

The rationale behind the conventional treatment of current induced spin torque is the following. The transmission of the spin current across the normal metal/ferromagnetic (N/F) interfaces is described by spin dependent reflection and transmission scattering amplitudes, and the Stoner model of spin split bands for the magnetic layers has provided a good description of electron transport in collinear magnetic multilayers and provides an explanation for one of the origins of giant magnetoresistance, spin dependent interface scattering due to differences in band structure at the N/F interfaces; the model does not involve spin flips and indeed provides a basis for understanding why spin-flips, which are high energy excitations, are rare ( impurity induced). This same model has been applied to noncollinear structures in which the angle between the magnetic layers is different from $0^{0}$ and $180^{\circ}$. One again with no spin flip scattering at the interfaces and by using the same parameters for the spin dependent interface scattering one finds the spin currents that were continuous in the collinear case now have discontinuities at the interfaces. 8] Indeed in this description these discontinuities represent the transfer of spin angular momentum from the current to the magnetic layer and thereby create a "spin torque" which eventually leads to current induced magnetization reversal. 1] The origin of the discontinuity in the spin current can be traced back to the transmission and reflection coefficients at $\mathrm{N} / \mathrm{F}$ interfaces. In this view the spin angular momentum lost by the spin current goes to the background magnetization which implies that a steady state for the spin current is not achieved before the background moves in such a manner as to remove the discontinuity in the spin current, which is of the order of a nanosecond.

An alternate view has been proposed in which a steady state spin current is achieved on the time scale of the longitudinal spin flip time, which is of the order 
of a picosecond. 9, 10] The salient difference in the two views is that we find that the discontinuity in the spin current at the interfaces drives a transverse spin accumulation which in turn achieves a steady state distribution for the spin current; in this manner the discontinuity is counterbalanced by accumulation so that $\partial_{t} f(k, r, t)=0$. In the conventional treatments one has not considered this transverse accumulation, rather one solved the transport equations of motion by setting $\partial_{t} f=0$, and thereby assumed steady state; if one inquires about the time to achieve this solution one would come up with a nanosecond. We have solved the time dependent equations of motion for transport across a noncollinear $\mathrm{F} / \mathrm{N} / \mathrm{F}$ structure using the same method we used for collinear structures; [5] the new ingredient is the transverse components of the discontinuity in the spin current. For thin normal layers, $10 \mathrm{~nm}$, it takes of the order of several femtoseconds for the spin accumulation created at one $\mathrm{N} / \mathrm{F}$ interface to reach the other interface. When the layers are noncollinear the longitudinal accumulation created by the first interface has a component transverse to the magnetization of the second magnetic layer. We find this drives a transverse accumulation of spin in the second magnetic layer which leads to a steady state solution in which the spin current is continuous across the interfaces. 11] In the presence of differences in band structure across interfaces it is necessary to include the current driven spin flip scattering at interfaces to inject a spin current with a component transverse to the magnetization of a ferromagnetic layer; if one limits oneself to the equilibrium transmission coefficients there is no excitation of the transverse spin current across an interface and thereby no transverse accumulation, i.e., the steady state solutions with and without the interface spin flip scattering are indeed different. For example we have found the resistivity for noncollinear structures is always lower when we consider this additional scattering. 12

To exhibit the main differences in our approach compared to the conventional one we have solved for electron transport in a noncollinear magnetic trilayer $\mathrm{F} / \mathrm{N} / \mathrm{F}$ by using the semiclassical Boltzmann equation of motion; we assumed that the nonmagnetic spacer is thin compared to the spin diffusion length so that one can effectively reduce the transport calculation that of two noncollinear ferromagnetic layers with transmission coefficients given in terms of the $\mathrm{N} / \mathrm{F}$ transmission amplitudes of the trilayer. 13. We take spin split but otherwise free electron bands; this is sufficient to model the band mismatch in the $3 \mathrm{~d}$ transition-metal ferromagnets. Parenthetically, we have derived the equations for a Fermi sea of electrons; when we neglect the current driven corrections to the Fermi sea and focus only on the Fermi surface (see below) our equations are the same as those we find by using the $s-d$ model. 10] In each layer the energy and density vary slowly on the length scale of the Fermi wavelength so that we can limit ourselves to the first term in the gradient expansion of the equation of motion. [14] When we limit ourselves to linear response in the external electric field we find the equations of motion for the elements of the spinor density matrix for each momentum state on the Fermi surface $k_{p}$ are, 15

$$
\partial_{t} f_{p}+v_{p}^{x} \partial_{x} f_{p}-e E v_{p} \delta\left(\varepsilon-\varepsilon_{F}\right)=-\frac{f_{p}-\left\langle f_{p}\right\rangle}{\tau_{p}}-\frac{f_{p}-\left\langle f_{p^{\prime}}\right\rangle}{\tau_{s f}},
$$


and

$$
\partial_{t} f_{p}^{ \pm}+v_{p}^{x} \partial_{x} f_{p}^{ \pm} \mp i \frac{J_{p}}{\hbar} f_{p}^{ \pm}=-\frac{f_{p}^{ \pm}-\left\langle f_{p}^{ \pm}\right\rangle}{\tau_{p}},
$$

where we have used a simplified index $p$ to denote the momentum $k_{p}$ of a state on the $n^{\text {th }}$ sheet of the Fermi surface(we suppress this index), $p^{\prime}$ are states of opposite spin to $p$, the average $\left\langle f_{p}\right\rangle$ represents elastic scattering to all states on the Fermi surface, $v_{p}^{x}$ is the component of the Fermi velocity along the electric field $E, J$ is the magnetic part of the energy and we have limited the current induced variations of the distribution function to those along the growth direction of a multilayered structure $x$ (also the field direction). The diagonal elements $f_{p}=f_{\alpha \alpha}\left(k_{p}, x\right)$ represents the occupancy of the state $k_{p}$; in equilibrium it is given by the Fermi function so that only the spin state $\alpha$ that crosses the Fermi level is occupied while the other is zero and we do not consider it further. The off diagonal elements $f_{p}^{ \pm} \sim f_{\uparrow \downarrow}\left(k_{p}, x\right)$, which we call a current induced spin coherence, 16] represent coherences between the state $k_{p}$ on the Fermi surface and the states with opposite spin; these coherences occur when we drive a spin current across a $\mathrm{N} / \mathrm{F}$ interface. [7] The scattering terms include those for states of the same spin on the Fermi surface $\tau_{p}^{-1}$ as well as those between sheets of opposite spin $\tau_{s f}^{-1}$; as $\tau_{s f}^{-1} \ll \tau_{p}^{-1}$ we include the latter only to have well defined boundary conditions on our distribution functions.

The steady state $\left(\partial_{t} f_{p}=0\right)$ solutions for the longitudinal components $f_{p}$ are well know. [17] From Eqs.(11) and (2) we see that the electric field only creates out of equilibrium longitudinal components of the distribution functions; in a homogeneous magnetic layer there is no coupling to the transverse components $f_{p}^{ \pm}$. However when the spin current from one layer is injected into another noncollinear magnetic layer the transverse components can be excited provided one includes the current induced spin flip scattering at the interface; their inclusion removes the discontinuity in the spin current at the interfaces. When we neglect collisions (the rhs of Eq.(2)) the transverse solutions in steady state are $f_{p}^{ \pm}(x) \sim \exp \pm \mathbf{i}\left(J_{p} / \hbar v_{p}^{x}\right) x$; when we average this over the Fermi surface we find an interference between individual $p$ or $\mathbf{k}$ states so that the transverse components of the spin currents in the magnetic layers, $j_{x}^{ \pm}(x) \sim \int v_{x}(k) f^{ \pm}(k, x) d k$, can be fit to a form approximating an exponential decay $\sim \exp -x / \lambda_{t r}$. In the ballistic regime $\lambda_{t r}=d_{J} \equiv h \overline{v_{F} / J}$ where the bar denotes an average over states on the $n^{t h}$ sheet of the Fermi surface under consideration; [15] while for diffusive systems where we consider the collision terms we find $\lambda_{t r}=\lambda_{J} \equiv \sqrt{d_{J} \lambda_{m f p} / 3 \pi}$ when using the spin diffusion equation. [10] Therefore when components of the spin current are injected into a magnetic layer that are transverse to its magnetization, we find they propagate a distance $\lambda_{t r}$ before decaying; as this distance is an order of magnitude greater than the Fermi wavelength one can describe the transverse spin currents in the semiclassical Boltzmann approach. As $\lambda_{t r}$ is comparable to the thickness of the magnetic layers undergoing switching one cannot assume the transverse components of spin currents are entirely absorbed in such thin layers.

The salient results we find from the steady state solutions of the above equations 

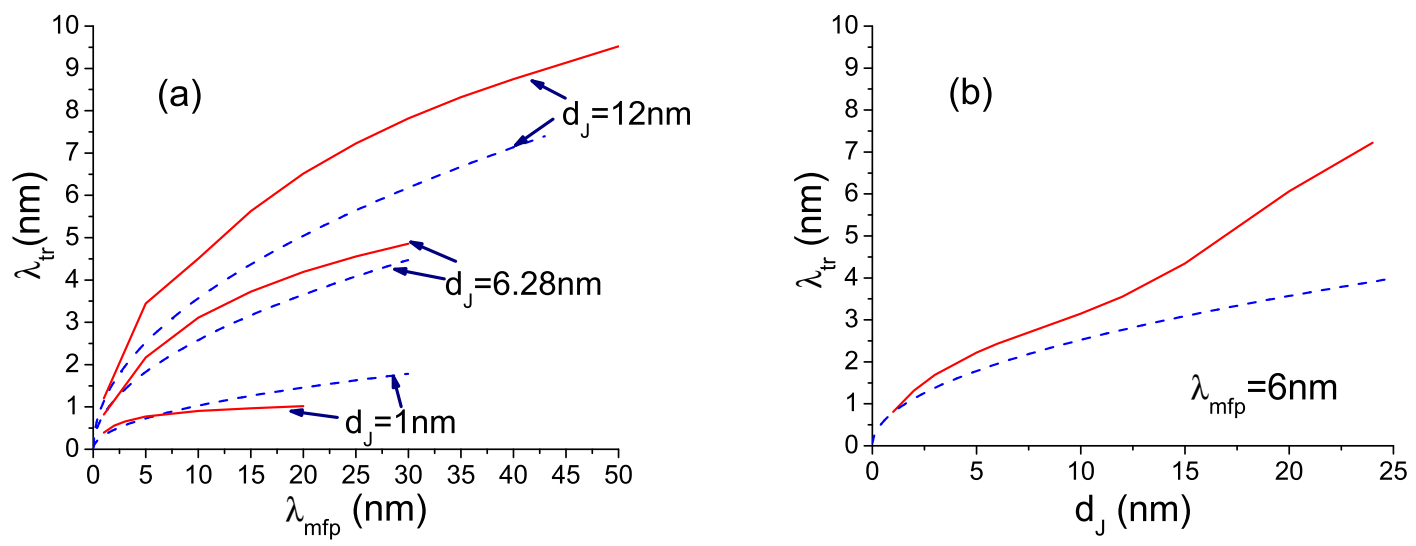

Figure 1. The transverse length of spin current $\lambda_{t r}$. The solid lines are the Boltzmann result, the dash lines are the diffusion result. In fig(a) we show the variation as a function of mean free path for different $d_{J}$; in (b) the variation with $d_{J}$ for a mean free path equal to $6 \mathrm{~nm}$.

are: transverse spin current and accumulation exist in the magnetic layers up to $\lambda_{t r} \sim 3$ $\mathrm{nm}$ from the interface in the ballistic regime, there is no discontinuity in the spin current provided one has included the spin flip at the interface, and when we include diffuse scattering in the layers $\lambda_{t r}$ is by and large different from the $\lambda_{J}$ found from the spin diffusion equation. 9] In Fig.1 we show the variation of the transverse decay length $\lambda_{t r}$ as a function of the exchange splitting $J$ and the mean free path $\lambda_{m f p}$; for the parameters that describe $C o$ we find the solutions found from the Boltzmann equation are reasonably well described by the spin diffusion equation in which one has neglected the spin splitting of the bands but included diffuse interface scattering as a resistance. 9] In these calculations the coherence between states of opposite spin are kept intact, because we have not used a spin polarized effective one electron description of the bands in the ferromagnetic layers. When we use the spin split band description we are able to retrieve the hallmark of the coherence, i.e., continuity of spin current across the interface, if we include the spin flip scattering coefficients at the interfaces; by using only the equilibrium transmission coefficients we find the spin current is discontinuous. We conclude that for spin transport in noncollinear multilayers it is more important to keep track of the spin coherence between states of opposite spin in ferromagnetic layers than the precise details of the spin split band structure.

We have also started to determine the time dependent solutions for the transport equations in noncollinear structures.[11] Initially there is a discontinuity in the spin current at the interfaces; the longitudinal component of this discontinuity is relaxed by the random spin flip scattering in the bulk of the layers. [5] The transverse components are relaxed by the third term on the left hand side of Eq.(2); however if one does not include the spin flip scattering at the interfaces the transverse components of the distribution function are not excited and there is no relaxation, i.e., one does not have 
a steady state solution, rather the discontinuity in the transverse component of the spin current remains until the free magnetic layer switches. We have also calculated the resistance of noncollinear trilayers (modelled as bilayers), and always find their resistance is lower when we include the interface spin flip scattering. [12]

In conclusion the steady state arrived at by using only the equilibrium transmission coefficients for noncollinear magnetic multilayers has different attributes from the one reached when either one uses the current induced interface scattering, or neglects the spin splitting of the band structure. They differ in their resistances, length scales of spin transfer, magnitudes of spin torque created, and the time to reach steady state. Specifically, providing all other things are equal, the spin torque in our model is less than that found in the conventional ones when the thickness of the layer to be switched is less than the transverse decay length which is of the order of 2-3 nm. These differences should be within the reach of current experimental conditions to ascertain which picture better fits the data.

We would like to thank Professor Shufeng Zhang for very helpful discussions. Research supported by the National Science Foundation under Grant DMR 0131883.

[1] J.C. Slonczewski, J. Mag. Mag. Mater. 159, L1 (1996); ibid 195, L261 (1999); L. Berger, Phys. Rev. B 54, 9353 (1996); J. Appl. Phys. 89, 5521 (2001).

[2] Kuising Wang, S. Zhang, and P.M. Levy,Phys. Rev. B 54,11965 (1996); X. Waintal, E.B. Myers, P.W. Brouwer and D.C. Ralph, Phys. Rev.B 62, 12317 (2000).

[3] C.L. Kane, R.A. Serota and P.A.Lee, Phys. Rev.B 37, 6701 (1988).

[4] P.M. Levy,H.E. Camblong and S. Zhang, J.Appl. Phys. 75, 7076 (1994); H.E. Camblong, P.M. Levy and S. Zhang, Phys. Rev.B 51, 16052 (1995).

[5] Shufeng Zhang and P.M. Levy, Phys. Rev. B 65, 052409 (2002).

[6] See Electrons and Phonons by J.M. Ziman ( Oxford at the Clarendon Press, London 1972); see pp. 275-285.

[7] Peter M. Levy and Jianwei Zhang, Current induced spin flip scattering at interfaces in noncollinear magnetic multilayers, submitted for publication.

[8] A. Brataas, Yu.V. Nazarov, and G.E.W. Bauer, Phys. Rev. Lett. 84, 2481 (2000) and D.H. Hernando, Y.V. Nazarov, A. Brataas, and G.E.W. Bauer, Phys. Rev.B 62, 5700 (2000); Alexey Kovalev, Arne Brataas and Gerrit E.W. Bauer, ibid 66, 224424 (2002); M.D. Stiles and A. Zangwill, Phys. Rev. B 66, 014407 (2002); M.D. Stiles and A. Zangwill, J.Appl. Phys. 91, 6812 (2002); J.C. Slonczewski, J. Mag. Mag. Mater. 247, 324 (2002); Gerrit.E.W. Bauer, Yaroslav Tserkovnyak, Daniel Huertas-Hernando and Arne Brataas, Phys. Rev. B 67, 094421 (2003).

[9] A. Shpiro, P.M. Levy and S. Zhang, Phys. Rev.B 67,104430 (2003).

[10] S. Zhang, P.M. Levy and A. Fert, Phys. Rev. Lett. 88, 236601 (2002).

[11] Jianwei Zhang and Peter M. Levy, Time dependent solutions of transport in noncollinear magnetic multilyers, in preparation.

[12] Jianwei Zhang and Peter M. Levy, The steady state in noncollinear magnetic multilayers, in preparation.

[13] X. Waintal, E.B. Myers, P.W. Brouwer and D.C. Ralph, Phys. Rev.B 62, 12317 (2000); Gerrit E.W. Bauer,Yaroslav Tserkovnyak, Daniel Huertas-Hernando and Arne Brataas, Adv. in Solid State Physics, 43, 383 (2003).

[14] J. Rammer and H. Smith, Rev. Mod. Phys. 58, 323 (1986); also see Transport Phenomena by H. Smith and H.H. Jensen; in particular Sec. 1.13.

[15] Jianwei Zhang, Peter M. Levy, Shufeng Zhang and Vladimir Antropov, Identification of transverse spin currents in noncollinear magnetic structures, submitted for publication. Jianwei Zhang, P.M. Levy and Shufeng Zhang, Bull. Amer. Phys. Soc. 48, 117 (2003); P.M. Levy, Jianwei 
Zhang and Vladimir P Antropov, ibid 48, 821 (2003).

[16] The off diagonal elements of a density matrix $\left|\phi_{a}><\phi_{b}\right|$ refer to coherences between states; only the diagonal elements $a=b$ represent the occupancy of a state.here we are interested in those induced by currents across noncollinear magnetic structures at the Fermi surface.

[17] T. Valet and A. Fert, Phys. Rev. B48, 7099 (1993). 